Aufhebungen von Verurteilungen und Herabsetzungen von Kaurionen für die revolutionäre Bewegung erkennen, deren personelle Schlagkraft dadurch erhalten wird...*

III.

In einem Erwiderungspapier hält Kinoy Lubell vor, er habe mit der Verdammung seiner Arbeit als *rcformistisch « eine „Etiketren-Jurisprudenz * betrieben. Lubell babe verkannt, daß es ihm gerade darauf angekommen sei, stets beide Extreme der gegenwärrigen Widersprüche zu erfassen, während Lubell diese Widersprüche ignoriere und immer nur eine Seire sehe. Es gehe darum, die wachsende Radikalisierung von Millionen Amerikanern und die Tendenz der Herschenden zu offen terroristischer Diktatur sowie die Zusammenhänge $z$ wischen beidem zu erkennen.

Kinoy setzr sich gegen die Implikation von Lubells Position zur Wehr, der Kampf gegen die Tendenz der Herrschenden, von der bürgerlichen Demokrarie zum Faschismus überzugehen, sei notwendig $»$ reformistisch $*$. Aber:

* Es ist wahr und muß offen zugegeben werden, daß der methodische Ansatz der Linken hier und im Ausland zur Entwicklung einer Strategie gegen die faschistische Bedrohung schwerwiegende Schwächen hatre. Diese manifestierten sich oft in einem "reformistischen Abgehen von den Zielen nationaler Befreiung und dem Ubergang vom Klassenkampf zu einer Politik, die unvermeidlich zur Absicherung des Kapiralismus und seiner politischen und ökonomischen Institurionen führte. Diese Schwächen waren in den Vereinigten Staaten besonders evident und endeten nicht selten darin, die Führung an den pluralistisch-intervencionistischen (liberal-corporare) Flügel der herrschenden Klasse abzugeben, um damir seinen politischen Arm, die Demokratische Partei, zu stärken. Damit wurde hartnäckig die schreiende Notwendigkeit mißachtet, eine antikapitalistische Massenbewegung zu schaffen, die den Widerstand gegen die Bestrebungen des reaktionärsten Flügels des Kapitals zum Ubergang zu faschistischen Herrschaftsmitteln zu organisieren und zu führen und gleichzeitig große Volksmassen zu lehren und durch den Kampf auf die einzige dauerhafte Antwort auf die faschistische Gefahr vorzubereiten hätte - die Ubernahme der Macht über die ökonomischen, sozialen und politischen Institutionen des Landes durch das Volk ....

Ubersetzung und Bearbeirung: Jan Geblsen

\title{
Falsche erste Schritte zur integrierten Juristenausbildung
}

\section{I.}

Die Unzufriedenheir mit dem unverbundenen Nacheinander von $\gg$ theorerischem* Universitätsstudium und *prakrischem* Vorbereitungsdienst scheint die ganze Juristenwelt erfaßt zu haben, wenn man den Deutschen Juristentag für einen einigermaßen verläßlichen Indikator halten darf. Je größer die Einigkeit über die Notwendigkeit einer neuen Verbindung von Theorie und Praxis wird, 
desto wahrscheinlicher leben in dem verbalen Modernismus die fundamentalen Gegensätze fort, die sich aus der verschiedenen Sicht des Verhältnisses von Theorie und Praxis ergeben.

Man sollte sich niche durch die "Experimentierklausel«, die $\$ sa Richtergesetz bringen soll, zu dem Glauben verleiten lassen, nun könne in aller Ruhe dic Spreu vom Weizen gesondert werden, um dann - nach ro Jahren Erfahrungen - vorurteilsfrei zu entscheiden. Man würde dann erstens verkennen, daß der Versuch einer sozialen Veränderung weder steucrbar noch rüdknehmbar ist wie ein naturwissenschaftliches Laborexperiment, sondern daß die beteiligten sozialen Institutionen, bei der Juristenausbildung also vor allem Universität und "Praxis" (d.h. nach wie vor Justiz), dabei um Einfluß und Selbstbestätigung kämọfen und somit mehr als restrikrive Bedingungen der in den Programmen beabsichtigten Veränderung wirken werden. Z weitens ist durchaus erlaubr zu bezweifeln, ob die Ermächtigung zum Experiment überhaupt ernst gemeint ist. Liest man doch schon heute in dem Fragenkatalog, mir dem der Direktor des wissenschaftlichen Dienstes des Bundestags, Professor Quaritsch, die rundreisenden Berichterstatter des Bundestagsrechtsausschusses für die Änderung des Richtergesetzes, die Abgeordneten Dichgans und Beermann, ausgestattet hat, folgende Fragen: * Muß sichergestellt werden, daß die Experimente nach Ablauf der rojährigen Erprobungszeit auf ein bundeseinheitliches Modell zurückgeführt werden können? Wie soll das geschehen? durch Beschränkung auf ein Grundmodell? ... oder wie sonst? «Schließlich scheint der gegenwärtige Allzwedspopanz, an den Universitäten drohe eine linke Machtergreifung, die Abgeordneten von der ruhigen Einsicht abzuhalten, daß jede Studienreform zunichte wird, wenn die Examina unbeeinflußt von der Reform und außcrhalb der Universität bleiben. Von diesen Problemen wird hier nichr die Rede sein, sondern von dem Zusammenhang zwischen Ausbildungsreform und Personalstruktur.

Die vorhandenen Differenzen zwischen den drei Konzepten für eine einphasige Juristenausbildung (Loccum, Hamburg/Deutscher Juristentag, ASJ) - sie liegen weniger in der Zielsetzung (alle erklären sich für eine wissenschaftlich-kritische Auf- und Vorbereitung der Berufspraxis), durchaus aber in der Durcharbeitung der Konzepte - könnten nämlich samt den neuen Inhalten belanglos sein gegenüber den Folgewirkungen falscher erster Schritte zu ihrer administrativ-personellen Verwirklichung.

Die entscheidende Phase der Reform liegt also beim oder gar schon vor dem Beginn.

\section{II.}

Bei der Juristenausbildung ist die Gefahr der Selbstverhinderung des gestedkten Reformziels durch falsche administrativ-personelle Maßnahmen schon zur Wahrscheinlichkeit geworden, bevor noch die verschiedenen Konzepte offiziell zu Leitbildern erklärt worden sind. Sei es, daß naiver Reformeifer vorangetrieben hat, sei es, daß momentane Verlegenheirslösungen als Sprung nach vorn :crkauft werden, man sogar selbst daran glaubt oder einfach gar nichts denkt (man sollte nicht vorschnell eine böse Absicht unterstellen, denn das hieße immerhin ein Bewußtsein über das voraussetzen, was getan wird) -, jedenfalls wird dabei ein Teilaspekt der Reform isoliert in Praxis umgesetzt, und damit die Reform paralysiert. Der falsche, weil isolierte erste Sobritt ist die im Zuge der Reform notwendige und deshalb positiv bewertete stärkere Einbeziehung der 
Praktiker in die Universitüt, dic isoliert zur Ubernahme der Universitätsausbildung durch eine unveränderte Praxis fübrt. Während bis vor kurzem an den juristischen Fakultäten nur vereinzelt Lehraufträge "zur Ergänzung des Lehrangebors" an Praktiker vergeben wurden, sei es für Spezialgebiete oder, was schon eine Verlegenheirslösung war, für repetitorähnliche Examinatorien u. ä., haben nunmehr ein paar Fakultäten Teile der normalen Universitätsausbildung an Praktiker übertragen. Die Unsicherheit in der Rechtsform der Einbeziehung zeigt die Unüberlegtheit gut an, denn teils wurde die alte Form des Lehrauftrags beibehalten, teils hat ein Zusammenwirken von Universität und Dienstherrn der Praktiker dicse zu Assistenten im Nebenberuf gemachr, wobei anscheinend sowohl bei der Einstufung für die Nebentätigkeitsgenehmigung - nur Einkünfte aus wissenschaftlicher Tätigkeit sind nicht abzuführen - als auch bei faktischen Freistellungen recht großzügig verfahren wird. Von einem unter irgendeinem Gesichtspunkt überlegten Selektionsverfahren ist nichts bekannt. Auch die folgenden Beispiele sind rein zufällig bekannt geworden. Mangel an Publizität entspricht dem Bewußtseinsstand. So sind in Köln und Hamburg jeweils etwa 20 Praktiker in der Universitätsausbildung tätig und haben den etablierten Lehrkräften naturgemäß vor allem bei der 2 war wichtigen, aber belastenden Einschulung der Anfänger Arbeit abgenommen. In Gießen sind es nur vier Richter, die unter Aufrechterhaltung der Oberverantwortung der jeweiligen Ordinarien eine BGB-Ubung und Arbeirsgemeinschaften zur Grundvorlesung Strafrecht übernommen haben.

III.

Solche Beispiele werden zunächst als bloß quantitativ vermehrte Einschaltung der Praktiker in die Universitätsausbildung heruntergespielt. Doch zeigen gerade solche Argumente, daß das Bewußtsein über die qualitative Funktion dieses Einbruchs fehlt, weil der Zusammenhang mit der Entwicklung der Personalstruktur an der Universität unbekannt bleibt. Dort haben sich in den letzten Jahren folgende Tendenzen gezeigt: Der Charakter der Assistentenstelle als Nachwuchsstellung wurde durch die Ausweitung dieser Stellen zum »Mittelbau * aufgelöst. Mit der Vermehrung hat man zunächst ganz pragmatisch und vor allem billig mit dem Zuwadıs an Lehraufgaben durch die steigenden Studentenzahlen und der Notwendigkeit effizienterer Formen des Lehrens und Lernens in Kleingruppen fertig werden wollen. Da der Ausbau der Professorenstellen nicht annähernd mit der Ausweitung des Mittelbaus Schritt hielt, waren die Berufschancen des einzelnen Assistenten innerhalb der Universität sehr geschmälert und, da sich so etwas herumspricht, wurde der Verwalter $*$, der $*$ bloß 4 promovieren wollte, notwendig zum typischen Stelleninhaber. Folglich sank das Interesse an der Universität.

Man hätte daraus die Folgerung ableiten können, wie es das BAK-Konzepr und noch der erste Entwurf des Bundeshochschulrahmengesetzes vom 1. 7. 1970 taten, daß die Nachwuchsstellung durch ecite Berufschancen für Forschung und Lehre zu restituieren wäre. Stattdessen verstärkt sich der Trend, Forschung und Lehre zu trennen und den Nachwuchs für Forschung auf wenige elitäre Forschungseinrichtungen zu konzentrieren. Die Blindheit der bestehenden Fakultäten trügt dazu noch bei, wenn sie, wie mancherorts geschehen, im Zuge der Umstrukturierung des Personalaufbaus als Ersatz für die bisherigen Assistentenstellen Dozenten neucr Art als eine Art Habilitationsstipendien beantragen und 
gleichzeitig als Ersatz für den Mitrelbau, der faktisch weite Teile der Lehre (unter diskriminierenden, weil den tatsächlichen Verhältnissen nicht gerecht werdenden Bezeichnungen wie Unterricht oder unselbständige Lehre) übernommen hatte, die man sich natürlich nicht wieder selbst aufladen kann und will, bereitwillig für die bloße Lehrfunktion eine eigene Berufsgruppe zugestehen. Begonnen hat diese Entwicklung mit den Akademischen Räten, fortgesetzt wird sie heute konsequent mit der Einsicht, man müsse auch eine Laufbahn, also den Aufstieg in Dauerstellen bieten, die, um attraktiv zu sein, auch den Titel Professor einbringen müssen.

Gradmesser für den Unterschied zur alten forschungsbezogenen Lehrtätigkeit im Sinne ciner Einheit von Forschung und Lehre wird am deutlichsten die Höhe der Lehrverpflichtung sein. Statt von 6-8 Stunden spricht man von bis zu 20 Wochenstunden. Es ist im übrigen noch keineswegs ausgemacht, daß wenigstens die Assistenzprofessoren und alle Professoren alter Art weiter an der Einheit von Forschung und Lehre teilhaben sollen, wie sich unschwer an $\$ 43$ Abs. 3 des Regierungsentwurfs eines Hodıschulrahmengesetzes (BR-Drucksache 689/70) ablesen läßt. Die Auseinanderentwicklung der verschiedenen Berufskarrieren wird sich inhalelich daran zeigen, daß den Inhabern bloßer Lehrstellen, auf die Breite gesehen, bestenfalls noch die Orientierung über den außerhalb gefundenen Stand der Wissenschaft, aber nicht mehr die aktive Beteiligung am Wissenschaftsprozeß selbst möglich ist. Wird diese Berufsgruppe aber wesentlicher Träger der neuen, schon wegen der Kleingruppenveranstaltung lehr- und personalintensiven Juristenbildung, so fehle die notwendige administrativpersonelle Struktur für die Inhaltsneubestimmung der Wissenschaft. Das Ziel emanzipativ-kritischer Neudefinition auch der juristischen Inhalte wird dadurch verhindert, daß die organisatorische Basis für ein nur durch Forschungsintensivierung zu erreichendes Umdenken fehit.

Der berechtigte Horror vor dem numerus clausus wird das Seine dazu beitragen, die Argumente der Finanzminister, wie man die Kapazität am schnelisten und billigsten ausbaut (nämlich durch Lehrstellen mit hohen Lehrverpflichtungen), unschlagbar zu machen. Die Uberführung des bisherigen Nachwuchses und Lenkung der künttigen Ausbilder in diese neuen Lehrstellen wird einfach dadurch bewerkstelligt, daß die Stellenpläne vor allem solche Posten anbieten. Die Regel dürfte es nicht sein, daß die auf eine forschungsbezogene Lehrtätigkeit ausgerichtete Personengruppe auch unter diesen Belastungen einen kritischen Impetus entwickeit oder lange durchhält, erst recht nicht ihn sachlich erweitert. Bei Is Wochenstunden Unterricht läßt sich rein zeitlich kein Projekt entwickeln und durchführen, das zur kritischen Aufbereitung der Berufspraxis nach allen Modellen der einphasigen Ausbildung erforderlich wäre. Erst recht nicht läßt sich das Neue an Studenten vermitteln, die sich auf unveränderte Examina und Prüfer vorbereiten müssen.

IV.

Woher soll dann der hohe Personalbedarf der intensivierten Lehre gedeckt werden? Man hat zunächst an den Einsatz von Studenten und Graduierten als Tutoren gedacht, die anhand vorfabrizierter und quasi-autorisierter Lehrmaterialien die Kleingruppen übernehmen soliten. Eine gewisse Relation zwischen der ungefestigten Stellung des Ausführenden und der Bindung an Lehrmaterialien schien durchaus beabsichtigt. Doch haben mehrere Gründe diese tendenziell 
offen der Verschulung und damit auch Autorisierting von Lehrinhaleen zustrebende Entwidklung gebremst, nicht zuletzt wegen der gesellschaftspolitischen Ausridhtung der demnächst in Frage kommenden Jahrgänge der Studenten, die ihre Verwendung wim Sinne der Effizienz « ambivalent erscheinen ließ. Aud würde das berechtigre Argument von der Doppelbelastung genauso wie gegen den promovierenden Assistenten auch gegen graduierte und studentische Tutoren gerichtet werden können. Das Reservoir universitätsinterner Lehrkapazität ist folglich erschöpft, der Personalbedarf muß notwendig von außerbalb gededst werden.

Als Aushilfe bleibe nur die Abordnung von Personen aus der Praxis in die Lchre oder, anders gesagr, die Úbernahme der Ausbildung durch Personen, denen institutionell nicht die systematische Reflexion ihrer Praxis ermöglicht wird. Was wie eine Notlösung aussieht, ist zugleich die Patentlösung für die gesellschaftlichen Verhältnisse: Die Integration von Theorie und Praxis zur verändernden Praxis wird einseitig aufgelöst in die "Einübung in Praxis, wie sie ist (V. Kröning, KJ 1970/3I7), unabhängig vom guten Willen und den Fähigkeiten einzelner Beteiligter.

Was immer schöne Hochschulgesetze und Reformpläne an hoben und subjektiv allgemein geteilten Zielen verkünden, die wirklichen Reformmöglichkeiten werden von der administrativ-personellen Struktur bestımmt. Es ist hierfür ein schönes Beispiel, daß sich der Regierungsentwurf für das Hochschulrahmengeserz im Gegensatz zu früheren Entwürfen durch Schweigen nahtlos an jenen umstrittenen Beschluß der Kultusministerkonferenz vom I2. 3. 1970 - Zielvorstellungen für die Personalstruktur im Hochschulbereich - anpaßt, der einmal die * Bibel« der sich anscheinend konzeptionslos entwickelnden Hochschulverhältnisse genannt wurde und offen für die Reduzierung der Universität auf die Ausbildung eintritr, also die Trennung von Forschung und Lehre fordert und als Lehrkraft den "Richter im Hochschuldienst " einführr, wobei die Parallele zum * Studienrat im Hochschuldienst * an den pädagogischen Abteilungen und dessen Uberführung in eine Universitätskarriere (unter Beibehaltung der hohen Lehrverpflichtungen!) keinen Zufall darstell.

Es spricht für eine mangelnde Reflexion über die Verwirklichungsbedingungen von Reform, daß der kritische Ansatz bei den Plänen für eine einphasige Ausbildung so leicht $z u$ verdrehen ist, daß beim Wettrennen um den Ort, an dem * das Modell« zuerst durchgesetze wird, von der Integrierung von Theorie und Praxis weithin nur die »praxisorientierte Ausbildung « übrig geblieben ist. Sonst hätte z. B. der Gedanke an die Erprobung in einer von der örtichen hohen Richterbürokratie notwendig dominierten Neugründung überhaupt nicht aufkommen können. Wurden doch die Vorstellungen des Hauptakteurs für Kassel, Präsident Wannagat, über die Integration von Theorie und Praxis einmal unbeabsichtigt auf den Satz reduziert: "Das Gerichtswesen auf allen Ebenen in Kassel bietet hierfür geeignete Hochschullehrer und Ausbildungsmöglichkeiten.» (Informationen des Arbeitskreises Universität Kassel, Nr. 21/1970, S. 2). Ob Hamburg etwas darüberhinaus bietet?

Hansvolker Ziegler 